\title{
Autoavaliação da saúde por idosos atendidos em um centro ambulatorial de referência
}

Self-evaluation of the health by elderly attending in an outpatient referral center Autoavaliación de la salud para los ancianos atendidos en un centro ambulatorial de referencia

\author{
Kéury Guimarães PEREIRA ${ }^{(1)}$ \\ Patrick Leonardo Nogueira da SILVA ${ }^{(2)}$ \\ Maricy Kariny Soares OLIVEIRA ${ }^{(3)}$ \\ Mônica Antar GAMBA ${ }^{(4)}$ \\ Elaine Cristina Santos ALVES ${ }^{(3)}$ \\ Aurelina Gomes e MARTINS ${ }^{(3)}$
}

Recebido: 1 dez 2016 Revisado: 5 maio 2017 Aceito: 10 maio 2017

\section{Autor de}

correspondência: Patrick Leonardo Nogueira da Silva patrick_mocesp70@hotmail .com

Conflito de interesses: Os autores declaram não haver nenhum interesse profissional ou pessoal que possa gerar conflito de interesses em relação a este manuscrito.

\section{Resumo}

Os brasileiros vivenciam consideráveis mudanças demográficas e etárias em decorrência do aumento da expectativa de vida e acentuado envelhecimento populacional. Objetivouse identificar a autoavaliação da saúde por idosos atendidos em um centro ambulatorial de referência. Trata-se de um estudo descritivo, exploratório, com abordagem quantitativa. A amostra compreendeu 217 idosos. Utilizou-se como instrumento de coleta de dados um questionário estruturado, adaptado, reduzido e validado. A mesma foi realizada em fevereiro de 2013 . Os dados foram tabulados e apresentados em tabelas. $O$ tratamento se deu por meio de análise estatística descritiva simples. Observou prevalência do sexo feminino, insatisfação com a vida atribuída por problemas de saúde na qual a maior parte a considerava ruim ou péssima. Destes problemas, prevaleceu a hipertensão arterial sistêmica e dor no corpo. Foi referida a capacidade de realização das atividades de vida diária sem ajuda. Parte relatou satisfação com as atividades realizadas no tempo livre. Foi relatada carência de companhia. Faz-se necessário a criação de centros e grupos de interação e convívio social da terceira idade, junto às instituições de ensino superior do município de modo a prolongar a expectativa de vida, bem como melhorar a qualidade e satisfação pessoal.

Descritores: Saúde do idoso, Autoavaliação, Satisfação Pessoal. 


\begin{abstract}
Brazilians experience considerable demographic and age changes due to the increase in life expectancy and marked aging of the population. Aimed to identify the self-assessment of health by elderly people attending a referral outpatient center. This is a descriptive, exploratory study with a quantitative approach. The sample comprised 217 elderly. A structured, adapted, reduced and validated questionnaire was used as instrument of data collection. The same was done in February 2013. The data were tabulated and presented in tables. The treatment was by means of simple descriptive statistical analysis. Observed female prevalence, dissatisfaction with the life attributed by health problems in which the majority considered it bad or bad. Of these problems, systemic arterial hypertension and pain in the body prevailed. The ability to perform activities of daily living without help was mentioned. Part reported satisfaction with activities carried out during free time. Lack of company has been reported. It is necessary to create centers and groups of interaction and social interaction of the elderly, next to the higher education institutions of the municipality in order to prolong life expectancy, as well as improve quality and personal satisfaction.
\end{abstract}

Keywords: Health of the elderly, Self-assessment, Personal satisfaction.

\title{
Resumen
}

La experiencia brasileña considerables cambios demográficos y la edad debido al aumento de la esperanza de vida y el envejecimiento de la población aguda. Se objetivó identificar la percepción de la salud para los ancianos atendidos en un centro de derivación de pacientes ambulatorios. Se trata de un estudio descriptivo, exploratorio con enfoque cuantitativo. La muestra fue de 217 personas de edad avanzada. Se utilizó como instrumento de recolección de datos un cuestionario estructurado, adaptado, reducida y validado. La misma se llevó a cabo en febrero de 2013. Los datos se tabularon y se presentan en las tablas. El tratamiento se administra mediante estadísticas descriptivas simples. La prevalencia observada de la insatisfacción femenina con vida dada por los problemas de salud en el que la mayoría consideró que era mala o muy mala. Estos problemas, prevalecieron hipertensión y dolor en el cuerpo. Se refiere a la capacidad de realizar las actividades de la vida diaria sin ayuda. Parte informó de satisfacción con las actividades realizadas en el tiempo libre. Se informó de la falta de compañía. Es necesaria la creación de centros y la interacción del grupo y la vida social de las personas mayores, junto con las instituciones de educación superior de la ciudad con el fin de aumentar la esperanza de vida y mejorar la calidad y la satisfacción personal.

Palabras clave: Salud del anciano, Autoevaluación, Satisfacción personal.

\section{Introdução}

O Brasil tem vivenciado importantes mudanças no perfil demográfico e na estrutura etária da população devido à elevação da expectativa de vida e o acentuado envelhecimento populacional. Esse fenômeno apresenta-se como uma realidade não apenas no Brasil, mas em todo o mundo. Apesar do mesmo ser uma aspiração da sociedade, só pode ser considerado uma conquista se for possível agregar qualidade aos anos adicionais de vida. ${ }^{1,2}$ 
Envelhecer de maneira saudável depende da interação multidimensional entre saúde física, saúde mental, independência na vida diária, integração social, suporte familiar e independência econômica. Alguns fatos comuns, como a perda de um familiar, problemas econômicos, doenças incapacitantes, distúrbio mental, ou um acidente, podem, juntos ou isoladamente, comprometer a capacidade funcional de um indivíduo. ${ }^{3}$

Os idosos apresentam características específicas, portanto, o planejamento da assistência à saúde para essa faixa etária requer dos profissionais uma avaliação cuidadosa, através da coleta de informações que contribuam na identificação de problemas que envolvem o cuidado integral, favorecendo o alcance do bem-estar físico, mental e social dos idosos, baseadas não somente em seus direitos, mas em suas verdadeiras necessidades e nos fatores de risco à saúde. ${ }^{4}$ A garantia de acesso a serviços de saúde de qualidade para a população idosa apresenta-se como novo desafio. O conhecimento das necessidades da terceira idade, principalmente na comunidade, é importante para subsidiar o planejamento da atenção à saúde a essa faixa etária. ${ }^{5}$

As políticas públicas no país voltadas à atenção ao idoso devem focar em ações de saúde preventivas, na redução das desigualdades de renda e educação e na melhoria das instituições de saúde, a fim de que os índices de incapacidade funcional nessa população diminuam. Para isso devem ser considerados aspectos como a capacidade funcional, a necessidade de autonomia, de participação, de cuidado, de autossatisfação e ainda possibilitar a elaboração de novos significados para a vida na idade avançada, a fim de que os anos adicionais alcançados sejam vividos de forma plena e com qualidade. ${ }^{6,7}$

Embora diversos estudos evidenciem a associação entre medidas de saúde objetivas e medidas auto referidas de saúde, existem ainda poucos estudos que objetivam verificar a relação da percepção do estado de saúde da pessoa idosa com fatores socioeconômicos, demográficos, capacidade funcional e problemas de saúde, por exemplo. ${ }^{8,9}$ A autopercepção do estado de saúde pelo idoso desponta como indicador relevante de seu bem-estar, útil para avaliar suas necessidades de saúde e para predizer sua sobrevida. ${ }^{10}$ 
Sendo assim, objetivou-se identificar a autoavaliação da saúde por idosos atendidos em um centro ambulatorial de referência.

\section{Metodologia}

Artigo da monografia intitulada "Autoavaliação da saúde por idosos atendidos em um centro ambulatorial de referência" apresentado ao Departamento de Enfermagem da Universidade Estadual de Montes Claros - UNIMONTES. Montes Claros (MG), Brasil. 2013.

Trata-se de um estudo descritivo, exploratório, com abordagem quantitativa, realizado no Centro de Referência em Assistência à Saúde do Idoso - CRASI da cidade de Montes Claros, Minas Gerais (MG).

A amostra foi constituída por 217 idosos, considerando que a média mensal de atendimentos na instituição é de 500 atendimentos. Para definição desta amostra foi realizado cálculo amostral na qual se observou um nível de confiança de $95 \%$ e uma margem de erro de $5 \%$. O cálculo foi feito utilizando-se a fórmula de cálculo amostral abaixo:

$$
n=\frac{N \cdot Z^{2} \cdot p \cdot(1-p)}{Z^{2} \cdot p \cdot(1-p)+e^{2} \cdot(N-1)}
$$

Onde:

n - amostra calculada

$\mathrm{N}$ - população

Z - variável normal padronizada associada ao nível de confiança

p - verdadeira probabilidade do evento

e - erro amostral

Para a constituição da amostra foram adotados os seguintes critérios de inclusão: a) Ser atendido pelo CRASI no mês de fevereiro de 2013; b) Estar em plenas 
condições mentais e psicológicas para responder ao questionário; c) Concordar em responder ao questionário de pesquisa antes ou após a consulta. Critérios de exclusão: a) Apresentar alterações mentais ou psicológicas; b) Recusar em responder ao questionário de pesquisa antes ou após a consulta.

Após a definição do número de sujeitos e antes da realização da coleta dos dados, foram observados os critérios de inclusão descritos anteriormente.

Antes do início da coleta, o projeto de pesquisa foi apreciado e aprovado pela Diretoria do CRASI a qual assinou um Termo de Concordância da Instituição - TCI para participação em pesquisa e a todos os entrevistados foram dadas informações acerca dos objetivos do estudo, solicitada à confirmação da participação voluntária dos mesmos mediante a assinatura no Termo de Consentimento Livre e Esclarecido - TCLE. Foram garantidos a todos os participantes o sigilo, o anonimato e a privacidade.

A coleta dos dados foi realizada no mês de fevereiro de 2013, por meio de entrevista direta, seguindo um questionário estruturado o qual teve como referência o instrumento Brazilian Old Age Schedule - BOAS, que foi validado no Brasil por Veras. ${ }^{11}$ Este é um questionário multidimensional de saúde composto por 113 questões que abordam sete dimensões da saúde do idoso: saúde física; utilização de serviços médicos e dentários; atividades da vida diária - AVD; recursos sociais; recursos econômicos; saúde mental; e necessidades e problemas que afetam o entrevistado. Cada uma destas dimensões permite ao pesquisador explorar algumas das áreas consideradas importantes na vida dos idosos devido aos impactos causados.

Devido à grande extensão do instrumento BOAS, e a fim de atender apenas aos objetivos propostos neste estudo, optou-se por utilizar um questionário adaptado e reduzido abordando algumas questões da seção de informações gerais e das seções II, IV e VIII que contemplam respectivamente a saúde física, as AVD e as necessidades e problemas que afetam o entrevistado.

Os dados foram coletados nos turnos da manhã e/ou da tarde na sala de 
espera do CRASI antes das consultas. Aplicou-se uma média de 13 questionários por dia. Os dados coletados foram agrupados e posteriormente lançados em um banco de dados de modo a utilizar o Programa StatisticalPackage for the Social Sciences (SPSS $\left.{ }^{2}\right)$, versão 18.0, o que viabilizou a construção de tabelas para posterior análise. Optou-se por realizar a análise estatística descritiva simples.

O estudo obedeceu aos preceitos éticos estabelecidos pela Resolução n 466, de 12 de dezembro de 2012, do Conselho Nacional de Saúde - CNS, na qual regulamenta a pesquisa envolvendo seres humanos. O projeto de pesquisa foi apreciado e aprovado pelo Comitê de Ética em Pesquisa da Universidade Estadual de Montes Claros - CEP UNIMONTES, protocolo no 152.345/2012, Certificado de Apresentação para Apreciação Ética- CAAE, nº 09306112.0.0000.5146.

\section{Resultados}

Conforme os aspectos socioeconômicos e demográficos, observou-se prevalência do sexo feminino (75,2\%), pardo(a) (46,6\%), de outra cidade de MG (53,9\%), com idade entre 60-69 anos (53,4\%). A maior parte (50,6\%) sabe ler e escrever, Ensino Básico (Primário) completo (49\%), casado(a)/amasiado(a) (47,2\%), a maioriareside sozinhos (42,8\%) e contribuem nas tarefas diárias do autocuidado (96,4\%) (Tabela 1).

Tabela 1. Perfil socioeconômico e demográfico dos idosos atendidos no centro ambulatorial de referência. Montes Claros (MG), 2013

\begin{tabular}{|c|c|c|}
\hline Variáveis & n & $\%$ \\
\hline \multicolumn{3}{|l|}{ Sexo } \\
\hline Masculino & 54 & 24,8 \\
\hline Feminino & 163 & 75,2 \\
\hline \multicolumn{3}{|l|}{ Cor/Raça } \\
\hline Branco & 84 & 38,7 \\
\hline Preto & 32 & 14,7 \\
\hline Pardo & 101 & 46,6 \\
\hline Amarelo & 0 & 0 \\
\hline Indígena & 0 & 0 \\
\hline
\end{tabular}




\begin{tabular}{|c|c|c|}
\hline \multicolumn{3}{|l|}{ Naturalidade } \\
\hline Montes Claros & 89 & 41 \\
\hline Outra cidade de Minas Gerais & 117 & 53,9 \\
\hline Outro Estado & 11 & 5,1 \\
\hline \multicolumn{3}{|l|}{ Idade } \\
\hline 60-69 anos & 116 & 53,4 \\
\hline 70-79 anos & 67 & 30,8 \\
\hline Acima de 80 anos & 34 & 15,8 \\
\hline \multicolumn{3}{|l|}{ Leitura/escrita } \\
\hline Sim & 110 & 50,6 \\
\hline Não & 107 & 49,4 \\
\hline \multicolumn{3}{|l|}{ Escolaridade } \\
\hline Nenhuma & 90 & 41,4 \\
\hline Ensino Básico (Primário) & 106 & 49 \\
\hline Ensino Fundamental ( $\left.1^{\circ} \mathrm{Grau}\right)$ & 19 & 8,7 \\
\hline Ensino Médio ( $\left.2^{\circ} \mathrm{Grau}\right)$ & 2 & 0,9 \\
\hline \multicolumn{3}{|l|}{ Estado conjugal } \\
\hline Casado/amasiado & 102 & 47,2 \\
\hline Viúvo & 90 & 41,4 \\
\hline Divorciado/separado & 24 & 11 \\
\hline Solteiro & 1 & 0,4 \\
\hline \multicolumn{3}{|c|}{ Número de pessoas residentes no domicílio } \\
\hline Mora só & 22 & 10,1 \\
\hline Apenas 01 pessoa & 93 & 42,8 \\
\hline $2-4$ pessoas & 80 & 37 \\
\hline $5-10$ pessoas & 22 & 10,1 \\
\hline \multicolumn{3}{|c|}{ Ajuda nas tarefas diárias do autocuidado } \\
\hline Sim & 209 & 96,4 \\
\hline Não & 8 & 3,6 \\
\hline
\end{tabular}

Fonte: Elaboração própria a partir de dados coletados em campo (2013).

${ }^{*}$ Total maior de 32, aceite de mais de uma alternativa. ${ }^{* *} \mathrm{n}=217$

Quando questionados sobre a satisfação com a vida, 69,5\% relataram estar satisfeitos. Dos 30,5\% que referiam insatisfação, 57,7\% atribuiu essa autoavaliação negativa principalmente aos problemas de saúde enfrentados (Tabela 2). Ressalta-se que alguns idosos relataram mais de um problema relacionado à sua insatisfação com a vida em geral. 
Tabela 2. Principais motivos de insatisfação com a vida autorreferidos pelos idosos atendidos no centro de referência ambulatorial. Montes Claros (MG), 2013

\begin{tabular}{|c|c|c|}
\hline Variáveis & n & $\%$ \\
\hline \multicolumn{3}{|c|}{ Autoavaliação da satisfação com a vida } \\
\hline Satisfeito & 164 & 75,6 \\
\hline Insatisfeito & 53 & 24,4 \\
\hline \multicolumn{3}{|c|}{ Principais motivos de insatisfação com a vida } \\
\hline Problema econômico & 7 & 13,2 \\
\hline Problema de saúde & 38 & 71,6 \\
\hline Problema de moradia & 0 & 0 \\
\hline Problema de transporte & 3 & 5,6 \\
\hline $\begin{array}{l}\text { Conflito nos relacionamentos } \\
\text { pessoais }\end{array}$ & 9 & 16,9 \\
\hline Falta de atividades & 0 & 0 \\
\hline Outros problemas & 9 & 16,9 \\
\hline
\end{tabular}

Fonte: Elaborado pelos autores com dados da pesquisa (2013).

* $n=217$

Apesar da autoavaliação com a vida ter sido positiva, 49,7\% dos entrevistados consideraram sua saúde ruim e 42,4\% consideraram boa. Ao comparar a situação atual de saúde com a situação de saúde apresentada há cinco anos, a situação de saúde atual foi considerada pior por 65,8\% dos entrevistados. Quando comparada com pessoas de mesma faixa etária, observou-se uma autoavaliação considerada melhor por 46,5\% (Tabela 3).

Tabela 3. Perfil da autoavaliação da situação de saúde a nível geral, comparada há cinco anos e com pessoas da mesma idade. Montes Claros (MG), 2013.

\begin{tabular}{|c|c|c|}
\hline Variáveis & n & $\%$ \\
\hline \multicolumn{3}{|c|}{ Autoavaliação da situação geral de saúde } \\
\hline Ótima & 4 & 2,1 \\
\hline Boa & 92 & 42,3 \\
\hline Ruim & 108 & 49,7 \\
\hline Péssima & 13 & 5,9 \\
\hline
\end{tabular}

Autoavaliação da saúde comparada com os últimos cinco anos

$\begin{array}{lcc}\text { Melhor } & 26 & 12,1 \\ \text { Igual } & 48 & 22,1 \\ \text { Pior } & 143 & 65,8\end{array}$


Autoavaliação da saúde comparada às pessoas da mesma idade

$\begin{array}{lcc}\text { Melhor } & 101 & 46,5 \\ \text { Igual } & 65 & 29,9 \\ \text { Pior } & 51 & 23,6\end{array}$

Fonte: Elaborado pelos autores com dados da pesquisa (2013).

${ }^{*} \mathrm{n}=217$

Observou-se que 97,7\% relataram ter algum problema de saúde. Destes, $69,3 \%$ disseram que os problemas de saúde atrapalham na realização das AVD. Os problemas de saúde identificados com maior prevalência na fase senil foram a Hipertensão Arterial Sistêmica (HAS) com 57,1\%, Dor no corpo (40,5\%) e Osteoporose (17\%) (Tabela 4). Ressalta-se que um mesmo indivíduo pode apresentar mais de uma patologia associada. Sendo assim, neste estudo os participantes relataram ter mais de um problema de saúde.

Tabela 4 - Principais problemas de saúde enfrentados pelos idosos. Montes Claros (MG), 2013

\section{Variáveis}

n $\%$

Apresenta problemas de saúde

\begin{tabular}{lcc} 
Sim & 212 & 97,7 \\
Não & 5 & 2,3 \\
\hline
\end{tabular}

Interferência dos problemas de saúde na realização das AVD $(n=212)$

\begin{tabular}{lcc} 
Sim & 147 & 69,3 \\
Não & 65 & 30,7 \\
\hline Problemas de saúde enfrentados & & \\
HAS & 124 & 57,1 \\
Dor & 88 & 40,5 \\
Osteoporose & 37 & 17 \\
DM & 35 & 16,1 \\
Artrose & 30 & 13,8 \\
Cansaço & 21 & 9,7 \\
Cardiopatia & 13 & 6 \\
Doença de Chagas & 11 & 5 \\
AVE & 10 & 4,6
\end{tabular}

Fonte: Elaborado pelos autores com dados da pesquisa (2013).

* $\mathrm{n}=217 ; \mathrm{n}=212$ 
Apenas $16,1 \%$ relataram ter problemas nos pés que atrapalham a sua mobilidade como joanete, calos, dedos torcidos ou unha encravada; $57,6 \%$ referiram problemas em articulações dos braços, mãos, pernas ou pés; e ninguém relatou amputação de membros. Houve prevalência de quedas em idosos $(21,7 \%)$ nos últimos três meses, sendo que $36,1 \%$ deles precisaram de ajuda para se levantar. A maior parte dos idosos (59,5\%) fez uma autoavaliação negativa quanto a sua visão, mesmo com a ajuda de óculos. Destes, $71,3 \%$ consideraram que esse problema de visão atrapalha de modo a causar insatisfação. Quanto à audição, com ou sem a ajuda de aparelhos auditivos, 69,6\% avaliaram como sendo ótima ou boa. Dos que avaliaram negativamente a audição, $71,2 \%$ relataram não atrapalhar. Com relação ao estado geral dos dentes, 60\%, consideraram como bom estado, mas ainda assim 66,4\% disseram apresentar algum tipo de problema ao mastigar os alimentos. A incontinência urinária (IU) dentre os idosos foi apontada com uma alta prevalência (51,6\%) (Tabela 5).

Tabela 5 - Distribuição de idosos segundo a presença de problemas que afetam a saúde nos últimos três meses. Montes Claros (MG), 2013

\begin{tabular}{lcc}
\multicolumn{1}{c}{ Variáveis } & $\mathbf{n}$ & $\%$ \\
Problemas nos pés que inibem a sua motilidade & & \\
Sim & 182 & 83,8 \\
Não & 35 & 16,2 \\
\hline Problemas nas articulações & & \\
Sim & 125 & 57,6 \\
Não & 92 & 42,4 \\
\hline Queda nos últimos três meses & & \\
Sim & 47 & 21,7 \\
Não & 170 & 78,3 \\
\hline Levanta-se sozinho do chão $(n=47)$ & & \\
Sim & 30 & 63,8 \\
Não & 17 & 36,2 \\
\hline
\end{tabular}

Autoavaliação da visão 


\begin{tabular}{lcc} 
Ótima & 4 & 1,8 \\
Boa & 84 & 38,7 \\
Ruim & 123 & 56,7 \\
Péssima & 6 & 2,8 \\
\hline
\end{tabular}

Este problema de visão atrapalha $(n=129)$

\begin{tabular}{lcc} 
Sim & 92 & 71,3 \\
Não & 37 & 28,7 \\
\hline Autoavaliação da audição & & \\
Ótima & 14 & 6,4 \\
Boa & 137 & 63,1 \\
Ruim & 61 & 28,1 \\
Péssima & 5 & 2,4 \\
\hline
\end{tabular}

Este problema auditivo atrapalha $(n=66)$

\begin{tabular}{lcc} 
Sim & 47 & 71,3 \\
Não & 19 & 28,7 \\
\hline Autoavaliação do estado geral dos dentes & & \\
Ótima & 0 & 0 \\
Boa & 130 & 59,9 \\
Ruim & 83 & 38,2 \\
Péssima & 4 & 1,9 \\
\hline
\end{tabular}

Tem algum problema de dente que atrapalha mastigar os alimentos

\begin{tabular}{lcc} 
Sim & 73 & 33,7 \\
Não & 144 & 66,3 \\
\hline Apresenta IU & & \\
Sim & 112 & 51,6 \\
Não & 105 & 48,4 \\
\hline
\end{tabular}

Fonte: Elaborado pelos autores com dados da pesquisa (2013)

* $n=217 ; n=212$

Observou-se que a maior parte da amostra foi capaz de realizar as principais AVD sem ajuda: comer sua refeição (98,6\%), caminhar em superfície plana (98,6\%), tomar banho $(97,7 \%)$ e vestir-se $(95,4 \%)$. No tempo livre, as AVD mais realizadas eram receber visitas (88,5\%), assistir televisão $(85,7 \%)$ e ir à igreja (79,3\%). Destaca-se que apenas $16,6 \%$ referiram praticar esporte ou realizar alguma atividade física (AF). Foi questionado sobre a 
satisfação com a prática de AVD no tempo livre, e observou-se que a maioria (62,2\%) relatou estar satisfeito com a realização destas atividades. Os 37,8\% que relataram estar insatisfeitos atribuíram esta autoavaliação negativa principalmente a problemas com a saúde (61\%) e com a falta de motivação (18,3\%) (Tabela 6).

Tabela 6 - Perfil da autoavaliação dos idosos quanto as AVD e sua satisfação em tempo livre. Montes Claros (MG), 2013.

Variáveis $\quad$ n $\%$

AVD

Sair de casa utilizando um transporte

Sair de casa dirigindo seu próprio carro

Sair de casa para curtas distâncias

Preparar sua própria refeição

Comer sua refeição

Realizar atividades domésticas

Tomar os seus remédios

Vestir-se

Pentear seus cabelos

Caminhar em superfície plana

Subir/descer escadas

Deitar e levantar da cama

Tomar banho

Cortar as unhas dos pés

Ir ao banheiro em tempo

AVD no tempo livre

Ouve rádio

Assistir televisão

Ler jornal

Ler revistas/livros

Receber visitas

Ir ao cinema/teatro

Andar pelo seu bairro

Ir à igreja (serviço religioso)

Ir a jogos
$129 \quad 59,4$

$23 \quad 10,6$

$181 \quad 83,4$

$167 \quad 77$

21498,6

$155 \quad 71,4$

19690,3

$207 \quad 95,4$

21398,2

21498,6

$161 \quad 74,2$

21398,2

$212 \quad 97,7$

$119 \quad 54,8$

$180 \quad 82,9$

$90 \quad 41,5$

$186 \quad 85,7$

$50 \quad 23$

$63 \quad 29$

19288,5

$16 \quad 7,4$

$138 \quad 63,6$

$172 \quad 79,3$

$8 \quad 3,7$ 


$\begin{array}{lcc}\text { Prática de algum esporte/AF } & 36 & 16,6 \\ \text { Fazer compras } & 137 & 63,1 \\ \text { Sair para visitar os amigos } & 134 & 61,8 \\ \text { Sair para visitar os parentes } & 163 & 75,1 \\ \text { Sair para passeios longos (viagens) } & 45 & 20,7 \\ \text { Sair para encontro social ou comunitário } & 25 & 11,5 \\ \text { Costurar/bordar/tricotar } & 76 & 35 \\ \text { Jogar cartas/xadrez/jardinagem } & 38 & 17,5 \\ \text { Outros } & 15 & 6,9\end{array}$

Autoavaliação da satisfação com as AVD no tempo livre

$\begin{array}{lcc}\text { Satisfeito } & 135 & 62,2 \\ \text { Insatisfeito } & 82 & 37,8\end{array}$

Principais motivos de insatisfação com as AVD em tempo livre $(n=82)$

$\begin{array}{lcc}\text { Problema com custo } & 10 & 12,2 \\ \text { Problema de saúde } & 50 & 61 \\ \text { Problema motivacional } & 15 & 18,3 \\ \text { Problema de transporte } & 3 & 3,6 \\ \text { Outras razões } & 4 & 4,9\end{array}$

Fonte: Elaborado pelos autores com dados da pesquisa (2013).

As principais necessidades/carências referidas foram: companhia (33,2\%), econômica $(28,1 \%)$ e de saúde (26,7\%). Houve idosos que não relataram nenhuma carência e outros relataram mais de uma necessidade. Os problemas relatados pelos idosos como sendo os mais importantes foram problema de saúde $(41,5 \%)$ e preocupação com os familiares (26,7\%). É importante destacar que 17,5\% referiram não ter nenhum problema cotidiano de grande relevância (Tabela 7). 
Tabela 7 - Necessidades ou carências referidas por idosos e sua autopercepção de problemas cotidianos mais prevalentes. Montes Claros (MG), 2013

\begin{tabular}{lcc}
\multicolumn{1}{c}{ Variáveis / Descrição } & n & $\%$ \\
Principais necessidades ou carências referidas & & \\
\hline Econômica & 61 & 28,1 \\
Moradia & 13 & 6 \\
Transporte & 32 & 14,7 \\
Lazer & 31 & 14,3 \\
Segurança & 18 & 8,3 \\
Saúde & 58 & 26,7 \\
Alimentação & 4 & 1,8 \\
Companhia & 72 & 33,2 \\
\hline Autopercepção do problema cotidiano mais prevalente & \\
\hline Não relata problemas & 38 & 17,5 \\
Problema econômico & 11 & 5,1 \\
Problema de saúde & 90 & 41,5 \\
Medo de violência & 3 & 1,4 \\
Problemas familiares & 11 & 5,1 \\
Problemas de isolamento & 4 & 1,8 \\
Preocupação com familiares & 58 & 26,7 \\
Outro problema & 2 & 0,9 \\
\hline Fonte: Elaborado pelos autos com dados da pesquisa (2013). &
\end{tabular}

Fonte: Elaborado pelos autores com dados da pesquisa (2013).

\section{Discussão}

O envelhecimento populacional apresenta-se como uma realidade em todo o mundo. Essa transição demográfica e, consequentemente epidemiológica, gera grandes desafios para a saúde devido ao surgimento de novas demandas, relacionadas às condições vivenciadas pela população idosa, como a prevalência de doenças crônicas as incapacidades funcionais. Assim, é necessário conhecer e entender esse contexto para criar 
e adequar políticas sociais, particularmente aquelas que atendam àscrescentes demandas na área da saúde, previdência e assistência social. ${ }^{12}$

Em relação ao sexo, este estudo demonstrou que a maior parcela da população estudada pertence ao sexo feminino. A predominância de mulheres idosas em relação a homens idosos já havia sido apontada por vários estudos desenvolvidos no Brasil. ${ }^{13-6} \mathrm{~A}$ maior frequência do sexo feminino é consequência de uma maior expectativa de vida ao nascer para as mulheres, o que pode ser explicado, por exemplo, pela elevada prevalência de óbitos por causas externas no sexo masculino e da capacidade que as mulheres têm de suportar maiores dificuldades funcionais se comparadas aos homens. Assim, a maior sobrevivência das mulheres não significa que estas desfrutem obrigatoriamente de melhores condições de saúde. ${ }^{17}$

Houve predominância de idosos com faixa etária entre 60 e 69 anos, o que se assemelha a resultados encontrados em estudos na qual a maior parte dos idosos também se encontrava nesta mesma faixa etária. ${ }^{10,16} \mathrm{O}$ nível de escolaridade vem sendo apontado como um importante indicador social e também como um fator determinante do processo saúde-doença. Neste estudo observou-se uma predominância de idosos com nível primário de educação seguido de analfabetismo. Estes dados demonstram que a população estudada apresenta um precário nível de desenvolvimento socioeconômico e que esta vive em condições de vulnerabilidade social. ${ }^{18} \mathrm{~A}$ baixa escolaridade compromete o acesso às estratégias de educação em saúde, impedindo, assim, as mudanças e a adesão a comportamentos saudáveis, assim como a mobilização social para a melhoria das condições de vida. ${ }^{19}$

Os fatores intervenientes no processo de adesão a tratamentos podem ser analisados segundo características relacionadas ao indivíduo, tais como: sexo, idade, etnia, estado civil, escolaridade e nível socioeconômico; a doença: cronicidade, e ausência de sintomas; crenças, valores: como hábitos culturais e de vida; percepção da severidade do problema, desconhecimento, experiência com a doença, contexto familiar, conceito sobre 
saúde, doença, autoestima; tratamento: custos, efeitos farmacológicos indesejáveis, esquemas complexos, qualidade de vida (QV); acesso: instituição, política de saúde, acolhimento, distância, atendimento, tempo de espera, forma e tipo de orientações, relacionamento com a equipe de saúde, envolvimento e relacionamento interpessoal ${ }^{20}$

Observou-se que a maioria dos entrevistados desta pesquisa reside com uma pessoa ou com duas a quatro pessoas. Estudos ${ }^{15,16}$ demonstram que uma parcela importante dos idosos morava com apenas uma pessoa no domicílio. Outros autores ${ }^{21}$ afirmam que morar acompanhado revelou-se como um fator determinante para incapacidade funcional. Quando questionados sobre a satisfação com a sua vida, a maioria dos entrevistados se diz satisfeito. Estudos ${ }^{15,10}$ apontaram resultados semelhantes ao encontrado nesta pesquisa.

A satisfação na velhice tem sido associada frequentemente a questões de saúde, autonomia, socioeconômicas, de bem-estar e relacionamento com os familiares e amigos. $^{15,22,23}$ A parcela de idosos que referiu insatisfação com a vida foi questionada sobre os fatores que estão relacionados à insatisfação e os mesmos relataram que esta está relacionada principalmente a problemas de saúde os quais os impedem de desenvolver AVD; uma pequena parte relaciona a insatisfação com conflitos nos relacionamentos pessoais, outros problemas não especificados e ainda problemas de ordem econômica. Estes resultados podem ser corroborados em outro estudo ${ }^{15}$ na qual a saúde e a independência foram os principais fatores determinantes da insatisfação.

Ao comparar a saúde atual com a saúde há cinco anos, a maioria dos idosos referiu que considera o estado de saúde atual pior. Apesar deste resultado, quando solicitados a comparar seu estado de saúde com o das outras pessoas da mesma idade, a maioria considerou sua saúde melhor. A comparação com outros indivíduos que estão supostamente em condições piores que a sua, tem importante papel na avaliação e construção da realidade e no enfrentamento de eventos negativos. ${ }^{24}$ 
Sendo a saúde da população idosa multidimensional, é necessário avaliar as tendências em saúde para esta população. Entre os eventos mais importantes a serem estudados, encontra-se a prevalência de doenças e de condições crônicas autorreferidas e a capacidade funcional. As condições de saúde que os idosos referiram estar enfrentando foram principalmente a HAS, a dor no corpo e a osteoporose, e em menores proporções, o diabetes mellitus (DM) e a artrose. Utilizando os dados da Pesquisa Nacional por Amostra de Domicílios (PNAD), desenvolvida entre os anos de 1998 a 2008, identificou-se aumento na prevalência da HAS e do DM, redução significativa na prevalência de artrite ou reumatismo e melhoria na autoavaliação da saúde e da capacidade funcional. ${ }^{7}$

Ao comparar os valores encontrados neste estudo com os resultados do inquérito nacional de 2008, o último feito no país, realizado através da PNAD quanto à prevalência de doenças crônicas autorreferidas, observou-se que a taxa de HAS encontrada neste estudo foi superior ao encontrado no inquérito nacional; a prevalência de DM apresentou resultado semelhante ao encontrado; e a prevalência de artrose foi apontada com menor frequência. Pesquisa realizada no município de Guatambu, Santa Catarina (SC), descreve que os problemas de saúde mais relatados foram: HAS, lombalgia e artrite/artrose. ${ }^{25} \mathrm{Em}$ estudo de base populacional desenvolvido na cidade de Londrina, Paraná (PR), observouse que a maioria dos idosos queixou de dor crônica, sendo os locais mais prevalentes desta dor a região dorsal e os membros inferiores (MMII). ${ }^{26}$ A dor crônica pode impactar na QV dos indivíduos, limitando suas decisões e comportamentos. Fatores como o afastamento social, incapacidade física e funcional, dependência, alterações da dinâmica familiar, desequilíbrio econômico e desesperança encontram-se também associados a quadros de dor. ${ }^{27}$

É importante ressaltar que o acidente vascular encefálico (AVE) também foi apontado como problema de saúde pelos idosos e que este é uma complicação importante relacionada à falta de controle adequado dos problemas de saúde relatados com maior frequência. Autores ${ }^{8: 61}$ colocam que "as condições físicas não são suficientes 
para determinar a forma de o idoso perceber o seu estado de saúde. Entretanto, algumas condições patológicas e comportamentais contribuem para uma percepção negativa do estado de saúde". Em outro estudo realizado por este mesmo autor, a HAS esteve relacionada com uma percepção negativa da saúde, este afirma ainda, que a influência desta patologia na avaliação negativa da saúde pode ser explicada, em parte, pelo impacto da enfermidade no dia-a-dia das pessoas a qual exige controle pressórico adequado de mudanças no estilo de vida e do uso regular de medicamentos.

A ocorrência frequente de quedas na população idosa é umas das principais preocupações relacionadas à saúde, pois faz com que os indivíduos acometidos necessitem, em muitos casos, de cuidado permanente e continuado. ${ }^{28}$ As quedas no idoso estão relacionadas a fatores intrínsecos, como o uso de medicamentos, diminuição da força muscular e do equilíbrio, instabilidade da marcha, doenças agudas e crônicas, como a osteoporose e fatores extrínsecos, como ambientes com pouca luz, tapetes soltos, escadas sem corrimão, pisos escorregadios e móveis dispostos inadequadamente. ${ }^{29} \mathrm{~A}$ prevalência de queda encontrada neste estudo foi inferior se comparada aos resultados apresentados por outros estudos realizados no Brasil. Este evento descrito como muito comum entre os idosos é considerado um problema de saúde pública devido a sua relação com altas taxas de mortalidade, ao alto custo social e econômico decorrentes das lesões e por serem eventos preveníveis. ${ }^{30}$

Estudiosos analisaram as causas e consequências de quedas em idosos residentes em Ribeirão Preto, São Paulo (SP), observou uma frequência de quedas maior em mulheres do que em homens, e como consequências destas quedas identificou a redução na capacidade funcional. ${ }^{31}$ Foi associado às quedas a uma percepção ruim ou regular da saúde, comprometimento da visão e dificuldade para andar. ${ }^{30}$ As proporções encontradas no presente estudo relacionadas à autoavaliação negativa da visão foram semelhantes às encontradas em estudo realizado pelo autor citado acima. A queda pode trazer consequências físicas e psicológicas, como a síndrome do medo de cair novamente, 
dificuldades para caminhar, ansiedade, ajuda para realizar as AVD, depressão e perda da autonomia. $^{29}$

Quando questionados sobre a autoavaliação da audição a maioria dos entrevistados referiu apresentar uma boa audição. Já a visão, obteve uma percepção negativa pela maioria dos idosos. A visão e a audição apresentam uma relação com a habilidade comunicativa. Aproximadamente um quinto da população idosa apresenta problemas de comunicação. A comunicação, juntamente com a cognição, o humor e a mobilidade são elementos fundamentais para a independência e complexidade na realização das AVD. ${ }^{32}$ Com relação à saúde bucal, estudos constataram uma auto percepção positiva das condições bucais mesmo com a alta porcentagem de pessoas utilizando algum tipo de prótese, total ou parcial. ${ }^{33,14}$ Apesar desta pesquisa não ter abordado especificamente a utilização de próteses dentárias ou a perda de dentes, a maioria dos idosos avaliaram as condições gerais dos seus dentes como sendo boa.

Observou-se também uma alta prevalência de idosos com IU, o que pode ser corroborado por meio do estudo desenvolvido em Pelotas, Rio Grande do Sul (RS), na qual apontou também uma alta prevalência de IU e/ou fecal. ${ }^{34}$ Estes autores de são vistas erroneamente pela população em geral como um processo natural do envelhecimento. Um terço das pessoas acima de 65 anos apresentam algum grau de IU, e esta porcentagem aumenta em idosos institucionalizados e mulheres. ${ }^{35}$ Estudo multicêntrico realizado no município de São Paulo demonstrou uma prevalência de IU maior nas mulheres se comparadas aos homens. ${ }^{36}$ Inquérito realizado na cidade de Salvador/BA apontou que a IU é um dos principais problemas de saúde que acomete a população idosa, principalmente a do sexo feminino. ${ }^{37}$ Os idosos acabam não relatando esse problema por sentirem vergonha e por acreditarem que é uma condição inevitável. Assim, a IU pode interferir na QV da população idosa acometida, causando constrangimento e induzindo ao isolamento social e depressão. ${ }^{38}$ 
Mesmo sendo acometidos por problemas de saúde, os idosos podem continuar desempenhando os seus papeis sociais. O foco da saúde está fortemente relacionado à funcionalidade global dos indivíduos. É considerado saudável o indivíduo que é capaz de realizar suas atividades de forma autônoma, mesmo que tenha doenças. ${ }^{39 "}$ "A manutenção da capacidade funcional do idoso é fundamental para a preservação de sua saúde" . ${ }^{40: 763}$ Em seu estudo estes autores encontraram uma maioria de idosos independentes para a realização das AVD. A grande maioria dos idosos relatou independência na realização das AVD questionadas, resultado que pode ser corroborado por outras pesquisas nas quais a maioria dos entrevistados relataram conseguir realizar atividades como, comer a sua própria refeição, tomar seus remédios sozinhos, vestirem-se, pentearem seus cabelos, tomarem banho e caminharem em superfície plana sem auxílio. ${ }^{25,34,7}$

A maior parte dos entrevistados referiu capacidade para a realização algumas atividades instrumentais da vida diária (AIVD) sem a necessidade de ajuda de familiares, tais como tomar seus medicamentos, arrumar a casa/cama, subir e descer escadas e sair de casa utilizando algum tipo de transporte. Foram consideradas como atividades difíceis de serem realizadas sem auxílio de outra pessoa sair de casa utilizando um transporte e o corte das unhas dos pés. Estudos mostram como incapacidade mais frequente para as AIVD os deslocamentos que necessitavam da utilização de algum meio de transporte ${ }^{34}$. Um estudo mensurou a incapacidade funcional e utilizou a variável mobilidade física por meio da habilidade que os idosos tinham para subir ladeira ou escada. ${ }^{21}$ Eles consideraram esta capacidade como um indicador robusto do declínio funcional entre os idosos.

Estudo realizado em 13 distritos de Portugal com indivíduos com idade igual ou maior que 75 anos mostrou que apenas uma minoria dos indivíduos foi considerada como dependente. ${ }^{41}$ Segundo a Organização Mundial da Saúde (OMS) ${ }^{42}$, um dos componentes mais importantes para a manutenção de uma boa saúde é através do estilo de vida adotado pelas pessoas, os seja, pelas ações realizadas pelo indivíduo no seu dia a dia, como a alimentação e a AF. 
Ao ser questionado sobre a prática de AF uma minoria dos idosos relatou ser fisicamente ativo. Este é um dado preocupante, pois a AF é fundamental para a prevenção da obesidade, HAS, DM, depressão, osteoporose, déficit cognitivo e mortalidade prematura. É importante manter o idoso ativo, a fim de ampliar o convívio social e a estimulação corporal. ${ }^{43} \mathrm{~A}$ prática de $\mathrm{AF}$ é um importante meio para a manutenção da capacidade funcional dos idosos, devendo, portanto, ser estimulada ao longo da vida. Deve-se priorizar para esta população o desenvolvimento da capacidade aeróbica, flexibilidade, equilíbrio, resistência e força muscular de acordo com a individualidade de cada idoso. ${ }^{44}$ É necessário motivar o idoso a participar de ações que visem à ocupação do tempo livre, favorecendo, dessa forma, uma melhor QV. ${ }^{45}$

Em um estudo qualitativo, foi demonstrado que os idosos permanecem muito tempo no domicílio e as atividades de lazer se resumem em assistir televisão e esperar uma visita, demonstrando um isolamento doméstico. Estes mesmos resultados foram auferidos neste estudo onde as atividades relatadas como as mais desenvolvidas pelos entrevistados foram assistir televisão, receber visitas e frequentar a igreja/serviço religioso. $^{45}$

A religiosidade contribui de forma significativa para a satisfação com a vida, pois ajuda a lidar de forma mais positiva com as situações negativas do dia-a-dia e fornece suporte espiritual, emocional e social. ${ }^{46}$ A religião tem a finalidade de fornecer um conforto moral e preservar valores espirituais do ser humano, além de desmistificar a morte. A fé pode auxiliar nos tratamentos implementados, pois através dela o indivíduo se cuida espiritualmente e exercita o autocuidado, podendo assim proporcionar motivação e consequente melhora na saúde. ${ }^{45}$ Sobre as necessidades apresentadas pelos idosos, neste estudo, a necessidade de maior prevalência referida foi a de companhia, mesmo a maioria dos idosos morando com uma ou mais pessoas. Ter alguém por perto não é necessariamente estar acompanhado, estar só não necessariamente é sentir-se só. O sentimento de solidão não pode ser entendido apenas como a ausência de pessoas por 
perto, mas sim como a vivência de sentimentos de desamparo, abandono ou desvalia. Idosos que relacionam o envelhecimento com decadência, desamparo e solidão tendem a sofrer maiores perdas físicas e morais. ${ }^{47}$ Estudo publicado evidenciou a necessidade dos idosos de ter alguém com quem conversar. As autoras demonstraram a importância de compreender essa necessidade e criar alternativas para supri-la, mesmo que para isso seja necessário reinventar o plano terapêutico, prescrevendo, por exemplo, "a conversa" com o idoso. $^{45}$

O convívio social deve ser incentivado na terceira idade, como forma de prevenir o isolamento e a solidão, através da realização de atividades, que preencham seu tempo e contribuam para a melhoria de sua saúde física e mental. ${ }^{48}$ Identificou-se que quase a totalidade dos idosos não participam de atividades que aprimorariam o convívio social. ${ }^{49}$ Os autores afirmam que a participação dos idosos em atividades de lazer é fundamental para a aquisição de novos conhecimentos e para a melhoria das condições de saúde. Essa prática reduz os custos com internações hospitalares e com terapêuticas medicamentosas, uma vez que torna essa população mais ativa e mais saudável fisicamente e intelectualmente. Políticas sociais que facilitem a inclusão dos idosos em atividades de lazer e cultura, disponibilidade de espaços de socialização que propiciem com que os idosos experimentem novos modos de viver e uma participação ativa na comunidade poderia contribuir para um maior bem-estar e maior satisfação pessoal. ${ }^{10,19}$

\section{Conclusão}

Conhecer os principais fatores determinantes das condições e autopercepção da saúde da população que envelhece é fundamental para promover o seu bem estar e satisfação. Esta pesquisa mostrou que a população estudada é composta em sua grande maioria por mulheres, com idade entre 60 e 69 anos, de cor predominantemente parda, casadas, que estudaram até o ensino primário, naturais de cidades do estado de Minas 
Gerais incluindo a cidade de Montes Claros, que residem com apenas uma pessoa e contam com esta ou outro para ajudar nas tarefas diárias e de autocuidado. Este estudo mostrou que os idosos se autoavaliam como satisfeitos com a vida e quando insatisfeitos esta insatisfação relaciona-se principalmente com problemas de saúde, de relacionamento ou outros problemas.

Observou-se prevalência de condições de saúde que, apesar de não terem interferido na independência das idosas, foram colocadas por elas por atrapalharem no dia a dia ou serem motivos de desconforto, como a perda da acuidade visual, a IU e a dor. Houve grande prevalência de morbidades crônicas e degenerativas, como a HAS, o que constitui fator de risco para incapacidades funcionais e consequentemente perda da autonomia, podendo fazer com que as idosas se tornem menos ativas fisicamente e socialmente. Entretanto, apenas uma minoria das entrevistadas apresentou dependência para as AVD e até mesmo para realizar sozinhas AIVD, mesmo estas constituindo tarefas de maior complexidade. Apesar deste contexto, poucas idosas são fisicamente ativas ou participam de atividades de socialização, sendo que as atividades desempenhadas são em sua grande maioria domiciliares e passivas.

A maioria das idosas refere uma autoavaliação negativa da sua saúde, mesmo estando satisfeitas com a vida, o que pode confirmar a hipótese de que os determinantes da felicidade e da sensação de bem estar vão além das condições físicas. Relataram ainda que a saúde atual é pior do que a apresentada por elas há cinco anos e melhor quando comparada com outras pessoas com a mesma idade. Os resultados do presente estudo indicam a necessidade crescente de ações integradas e multiprofissionais que adotem um modelo preventivo e promovam o envelhecimento saudável e a manutenção da funcionalidade global. O incentivo educacional nesta faixa etária seria uma estratégia que além de melhorar a autoestima, favoreceria a eficácia da educação em saúde, que busca modificar os hábitos de vida para prevenção de doenças crônicas e promover a adesão a tratamentos e cuidados prescritos, reduzindo complicações como o AVE. 
Faz-se necessário também a criação de centros e grupos de interação e convívio social da terceira idade, junto às instituições de ensino superior do município, por exemplo, para que esta população tenha anos adicionais de vida com qualidade e satisfação pessoal. Os resultados desse estudo oferecem informações que podem subsidiar ações a serem desenvolvidas pelos gestores/profissionais de saúde. Mas como descreve a realidade de idosos atendidos em um centro de referência específico, fazem-se necessárias maiores investigações a respeito deste tema.

\section{Referências}

1. Veras RP. Envelhecimento populacional contemporâneo: demandas, desafios e inovações. Rev Saude Publica. 2009;43(3):548-54. http://dx.doi.org/10.1590/S003489102009005000025 .

2. Bulgarelli AF, Pinto IC, Rodrigues Junior AL, Manço ARX. Estudo das queixas sobre saúde bucal em uma população de idosos na cidade de Ribeirão Preto - SP. Rev Bras Geriatr Gerontol. 2009;12(2):175-91. http://dx.doi.org/10.1590/1809-9823.2009.12022.

3. Ramos LR. Fatores determinantes do envelhecimento saudável em idosos residentes em centro urbano: Projeto Epidoso, São Paulo. Cad Saude Publica. 2003;19(3):793-8. http://dx.doi.org/10.1590/S0102-311X2003000300011.

4. Pilger $\mathrm{C}$, Menon $\mathrm{MH}$, Mathias TAF. Características sociodemográficas e de saúde de idosos: contribuições para os serviços de saúde. Rev LatAm Enferm. 2011;19(5):[9 telas]. http://dx.doi.org/10.1590/S0104-11692011000500022.

5. Matos DL, Lima-Costa MF. Tendência na utilização de serviços odontológicos entre idosos brasileiros e fatores associados: um estudo baseado na Pesquisa Nacional por Amostra de Domicílios (1998 e 2003). Cad Saude Publica. 2007;23(11):2740-8. http://dx.doi.org/10.1590/S0102-311X2007001100021. 
6. Parahyba MI, Veras R. Diferenciais sociodemográficos no declínio funcional em mobilidade física entre os idosos no Brasil. Cienc Saude Colet. 2008;13(4):1257-64. http://dx.doi.org/10.1590/S1413-81232008000400022.

7. Lima-Costa MF, Matos DL, Camargos VP, Macinko J. Tendências em dez anos das condições de saúde de idosos brasileiros: evidências da Pesquisa Nacional por Amostra de Domicílios (1998, 2003, 2008). Cienc Saude Colet. 2011;16(9):3689-96. http://dx.doi.org/10.1590/S141381232011001000006.

8. Silva RJS, Smith-Menezes A, Tribess S, Rómo-Perez V, Virtuoso Júnior JS. Prevalência e fatores associados à percepção negativa da saúde em pessoas idosas no Brasil. Rev Bras Epidemiol. 2012;15(1):49-62. http://dx.doi.org/10.1590/S1415790X2012000100005.

9. Idler EL, Benyamini Y. Self-rated health and mortality: a review of twenty-seven community studies. J Health Soc Behav. 1997;38(1):21-37. http://dx.doi.org/10.2307/2955359

10. Jóia LC, Ruiz T, Donalísio MR. Grau de satisfação com a saúde entre idosos do município de Botucatu, Estado de São Paulo, Brasil. Epidemiol Serv Saude. 2008;17(3):187-94. http://dx.doi.org/10.5123/S1679-49742008000300004.

11. Veras RP. País jovem com cabelos brancos: a saúde do idoso no Brasil. Rio de Janeiro: Relume Dumará; 1994.

12. Instituto de Estudos de Saúde Suplementar. Envelhecimento populacional e os desafios para o sistema de saúde brasileiro. São Paulo: IESS; 2013.

13. Lima-Costa MF, Firmo JOA, Uchôa E. A estrutura da autoavaliação da saúde entre idosos: projeto Bambuí. Rev Saude Publica. 2004;38(6):827-34. http://dx.doi.org/10.1590/S003489102004000600011.

14. Lebrão ML, Laurenti R. Saúde, bem-estar e envelhecimento: o estudo SABE no município de São Paulo. Rev Bras Epidemiol. 2005;8(2):127-41. http://dx.doi.org/10.1590/S1415$790 \times 2005000200005$.

15. Jóia LC, Ruiz T, Donalisio MR. Condições associadas ao grau de satisfação com a vida entre a população de idosos. Rev Saude Publica. 2007;41(1):131-8. http://dx.doi.org/10.1590/S003489102007000100018. 
16. Borim FSA, Barros MBA, Neri AL. Autoavaliação da saúde em idosos: pesquisa de base populacional no município de Campinas, São Paulo, Brasil. Cad Saude Publica. 2012;28(4):76980. http://dx.doi.org/10.1590/S0102-311X2012000400016.

17. Instituto Brasileiro de Geografia e Estatística. Indicadores sociodemográficos e de saúde no Brasil. Rio de Janeiro: IBGE; 2009.

18. Instituto Brasileiro de Geografia e Estatística. Síntese de indicadores sociais: uma análise das condições de vida da população brasileira. Rio de Janeiro: IBGE; 2008.

19. Geib LTC. Determinantes sociais da saúde do idoso. Cienc Saude Colet. 2012;17(1):123-33. http://dx.doi.org/10.1590/S1413-81232012000100015.

20. Santos ZMSA, Frota MA, Cruz DM, Holanda SDO. Adesão do cliente hipertenso ao tratamento: análise com abordagem interdisciplinar. Contexto Texto Enferm. 2005;14(3):332-40. http://dx.doi.org/10.1590/S0104-07072005000300003.

21. Alves LC, Leite IC, Machado CJ. Fatores associados à incapacidade funcional dos idosos no Brasil: análise multinível. Rev Saude Publica. 2010;44(3):1-11. http://dx.doi.org/10.1590/S003489102010005000009 .

22. Moraes JFD, Souza VBA. Fatores associados ao envelhecimento bem-sucedido de idosos socialmente ativos da região metropolitana de Porto Alegre. Rev Bras Psiquiatr. 2005;27(4):3028. http://dx.doi.org/10.1590/S1516-44462005000400009.

23. FrieswijkN, Buunk BP, Steverink N, Slaets JPJ. The interpretation of social comparison and its relation to life satisfaction among elderly people: does frailty make a difference?. J Gerontol B Psychol Sci Soc Sci. 2004;59(5):P250-7.

24. Sposito G, Diogo MJDE, Cintra FA, Neri AL, Guariento ME, Sousa MLR. Relações entre o bemestar subjetivo e a funcionalidade em idosos em seguimento ambulatorial. Rev Bras Fisioter. 2010;14(1):81-9. http://dx.doi.org/10.1590/S1413-35552010000100013.

25. Santos K, Koszuosky R, Dias-da-Costa J, Pattussi M. Fatores associados com a incapacidade funcional em idosos do município de Guatambu, Santa Catarina, Brasil. Cad Saude Publica. 2007;23(11):2781-8. http://dx.doi.org/10.1590/S0102-311X2007001100025.

26. Dellaroza MSG, Pimenta CAM, Matsuo T. Prevalência e caracterização da dor crônica em idosos não institucionalizados. Cad Saude Publica. 2007;23(5):1151-60. http://dx.doi.org/10.1590/S0102-311X2007000500017. 
27. Cunha LL, Mayrink WC. Influência da dor crônica na qualidade de vida em idosos. Rev Dor. 2011;12(2):120-4. http://dx.doi.org/10.1590/S1806-00132011000200008.

28. Del Duca GF, Thumé E, Hallal PC. Prevalência e fatores associados ao cuidado domiciliar a idosos. Rev Saude Publica. 2011;45(1):113-20. http://dx.doi.org/10.1590/S003489102010005000047.

29. Fhon JRS, Wehbe SCCF, Vendruscolo TRP, Stackfleth R, Marques S, Rodrigues RAP. Quedas em idosos e sua relação com a capacidade funcional. Rev Lat Am Enferm. 2012;20(5):924-34. http://dx.doi.org/10.1590/S0104-11692012000500015.

30. Cruz DT, Ribeiro LC, Vieira MT, Teixeira MTB, Bastos RR, Leite ICG. Prevalência de quedas e fatores associados em idosos. Rev Saude Publica. 2012;46(1):138-46. http://dx.doi.org/10.1590/S0034-89102011005000087.

31. Fabricio SCC, Rodrigues RAP, Costa Junior ML. Causas e consequências de quedas de idosos atendidos em hospital público. Rev Saude Publica. 2004;38(1):93-9. http://dx.doi.org/10.1590/S0034-89102004000100013.

32. Brasil. Ministério da Saúde. Organização Pan-Americana de Saúde. Organização Mundial de Saúde. Atenção à saúde do idoso: aspectos conceituais. Brasília, DF: MS/OPAS/OMS; 2012.

33. Lima-Costa MF, Peixoto SV, Matos DL, Firmo JOA, Uchôa E. A influência de respondente substituto na percepção da saúde de idosos: um estudo baseado na Pesquisa Nacional por Amostra de Domicílios $(1998,2003)$ e na coorte de Bambuí, Minas Gerais, Brasil. Cad Saude Publica. 2007;23(8):1893-1902. http://dx.doi.org/10.1590/S0102-311X2007000800016.

34. Del Duca GF, Silva MC, Hallal PC. Incapacidade funcional para atividades básicas e instrumentais da vida diária em idosos. Rev Saude Publica. 2009;43(5):796-805. http://dx.doi.org/10.1590/S0034-89102009005000057.

35. Duarte MB, Rego MAV. Comorbidade entre depressão e doenças clínicas em um ambulatório de geriatria. Cad Saude Publica. 2007;23(3):691-700. http://dx.doi.org/10.1590/S0102$311 \times 2007000300027$.

36. Bolina AF, Dias FA, Santos NMF, Tavares DMS. Incontinência urinária autoreferida em idosos e seus fatores associados. Rev RENE. 2013;14(2):354-63.

http://dx.doi.org/10.15253/rev\%20rene.v14i2.3391. 
37. Higa R, Lopes MHBM. Fatores associados com a incontinência urinária na mulher. Rev Bras Enferm. 2005;58(4):422-8. http://dx.doi.org/10.1590/S0034-71672005000400008.

38. Busato Junior WFS, Mendes FM. Incontinência urinária entre idosos institucionalizados: relação com mobilidade e função cognitiva. Arq Catarin Med. 2007;36(4):49-55.

39. Moraes EN. Princípios básicos de geriatria e gerontologia. Belo Horizonte: Coopmed; 2009.

40. Paskulin LMG, Vianna LAC. Perfil sociodemográfico e condições de saúde auto-referidas de idosos de Porto Alegre. Rev Saude Publica. 2007;41(5):757-68. http://dx.doi.org/10.1590/S003489102007000500010.

41. Sousa L, Galante H, Figueiredo D. Qualidade de vida e bem-estar dos idosos: um estudo exploratório na população portuguesa. Rev Saude Publica. 2003;37(3):364-71. http://dx.doi.org/10.1590/S0034-89102003000300016.

42. Organização Mundial de Saúde. Centro de Investigação em Actividade Física, Saúde e Lazer. Actividade física e saúde na Europa: evidências para a acção. Porto: OMS/CIAFEL; 2006.

43. Benedetti TRB, Borges LJ, Petroski EL, Gonçalves LHT. Atividade física e estado de saúde mental de idosos. Rev Saude Publica. 2008;42(2):302-7. http://dx.doi.org/10.1590/S003489102008005000007.

44. Maciel MG. Atividade física e funcionalidade do idoso. Motriz. 2010;16(4):1024-32. http://dx.doi.org/10.5016/1980-6574.2010v16n4p1024.

45. Nunes LM, Portella MR. O idoso fragilizado no domicílio: a problemática encontrada na atenção básica em saúde. Boletim Saude. 2003;17(2):109-21. 20140521090152v17n2_14idosofragilizado.pdf.

46. Cardoso MCS, Ferreira MC. Envolvimento religioso e bem-estar subjetivo em idosos. Psicol Cienc Prof. 2009;29(2):380-93. http://dx.doi.org/10.1590/S1414-98932009000200013.

47. Verdi MT. Vínculos: antídoto da solidão. Rev SPAGESP. 2010;11(2):17-23.

48. Duarte YAO. Atendimento domiciliar: um enfoque gerontológico. São Paulo: Atheneu; 2000.

49. Peregrino AAF, Schutz V, Marta CB, Pereira ACA, Silva GP, Nogueira LC. Buscando a inserção dos idosos nas ações de promoção social e de saúde. Rev Enferm UERJ. 2012;20(4):513-8. 


\section{Minicurrículo}

Kéury Guimarães Pereira | ORCID: 0000-0003-3126-2997

Especialista em Saúde Cardiovascular (Residência Multiprofissional) pela Universidade Federal de Minas Gerais - UFMG. Enfermeira do Hospital Regional de Irecê.

Patrick Leonardo Nogueira da Silva | ORCID: 0000-0003-2399-9526

Especialista em Saúde da Família pela Universidade Estadual de Montes Claros - UNIMONTES. Enfermeiro da Prefeitura Municipal de Espinosa.

Maricy Kariny Soares Oliveira | ORCID: 0000-0002-1303-8513

Doutora em Ciências da Educação com ênfase em Psicanálise pela Universidade Evangelista do Paraguai e Cambridge - UEPC. Professora do Departamento de Enfermagem da Universidade Estadual de Montes Claros - UNIMONTES.

Mônica Antar Gamba | ORCID: 0000-0003-1470-4474

Doutora em Saúde Pública pela Universidade de São Paulo - USP. Professora associada do Departamento de Enfermagem da Universidade Federal de São Paulo - UNIFESP.

Elaine Cristina Santos Alves | ORCID: 0000-0003-1357-9341

Mestre em Ciências da Saúde pela Universidade Federal de São Paulo - UNIFESP. Professora do Departamento de Enfermagem da Universidade Estadual de Montes Claros - UNIMONTES.

Aurelina Gomes e Martins | ORCID: 0000-0003-3582-8994

Mestre em Saúde Pública pela Universidade Federal de São Paulo - UNIFESP. Professora do Departamento de Enfermagem da Universidade Estadual de Montes Claros - UNIMONTES. 